\title{
LA INFLUENCIA DE LOS RECURSOS HUMANOS EN LA VENTA LIBRE DE LA OFICINA DE FARMACIA
}

\section{THE INFLUENCE OF HUMAN RESOURSES IN THE SALE OF OVER-THE-COUNTER DRUGS OF THE PHARMACY}

\author{
Losada Pérez, Fernando (Universidad de Santiago de Compostela) * \\ Torres Labandeira, Santiago (Universidad de Santiago de Compostela) ${ }^{* *}$
}

\begin{abstract}
RESUMEN
Son numerosas las investigaciones realizadas en diferentes sectores empresariales que analizan la importancia que los recursos humanos tienen en el éxito de las organizaciones, aunque son muy escasos los trabajos realizados en el sector de las farmacias. Entre los aspectos analizados en estas investigaciones se encuentran: la orientación de los empleados a los clientes, la formación de los recursos humanos y los incentivos establecidos por las empresas para sus trabajadores. En este sentido, el objetivo del presente trabajo de investigación, de naturaleza exploratoria, es analizar a través de una encuesta personal una muestra de 234 oficinas de farmacia de Galicia, la influencia que estas variables ejercen sobre la venta libre (productos de parafarmacia y especialidades farmacéuticas publicitarias), ya que como consecuencia de los profundos cambios ocurridos en este sector en los últimos años, estas empresas han perdido un gran volumen de facturación en la venta de medicamentos con receta, lo que las debe impulsar a centrar parte de sus esfuerzos comerciales en la venta de este tipo de productos. Para la verificación de las hipótesis planteadas se utilizó una regresión logística ordenada y la tabulación cruzada (prueba chi-cuadrado). Los resultados obtenidos en este estudio confirman la importancia que una gestión de los recursos humanos de las oficinas de farmacia basada en la orientación hacia los consumidores, la formación de los empleados y la implantación de incentivos, tiene en la venta libre.
\end{abstract}

Palabras claves: recursos humanos, formación, incentivos, orientación al cliente, oficinas de farmacia. JEL: M12, M54.

\footnotetext{
ABSTRACT

There are numerous researches which analyze the importance of human resources in the success of the organizations, although there are very little studies done in the pharmacy sector. The aspects considered included the following: the employee customer orientation, the training of human resources and the employee incentives established by the companies. In this sense, the main purpose of this work, that has exploratory nature, is to analyse the influence of those variables on the sale of over-the-counter drugs (parapharmacy products and advertising pharmaceutical specialties). The empirical analysis is based on the information provided by 234 Galician pharmacies through a personal interview. Due to the changes which have taken place in this sector in recent years, these companies have lost a high volume of

* Departamento de Organización de Empresas y Comercialización, Facultad de Administración y Dirección de Empresas, Avda. Alfonso X O Sabio, s/n.|27002 Lugo. fernando.losada@usc.es.

** Departamento de Campus Lugo, Escuela Universitaria de Relaciones Laborales (Lugo), Complexo docente do campus de Lugo | 27002 Lugo.santiagotorres@farmacialabandeira.com.

Recibido: 18 de septiembre de 2017. Aceptado: 13 de abril de 2018.
} 
prescription drugs sales; for this reason, they should focus some of their commercial initiatives on the sales of over-the-counter drugs. An Ordinal Logistic Regression and the Cross tabulation (Chi-square test) were used to test the proposed hypotheses. The results of this study confirm the importance that a good staff management has in the sale of over-thecounter drugs in the pharmacy, which is based on the customer orientation, the training of employees and the introduction of salary incentives.

Keywords: human resources, training, incentives, customer orientation, pharmacy. JEL: M12, M54.

\section{INTRODUCCIÓN}

Las oficinas de farmacia en España se encuentran ante uno de los mayores desafíos a los que se hayan enfrentado en las últimas décadas, motivado por la coyuntura económica que vive el país, el cambio en los hábitos de compra de los consumidores y los cambios legislativos promovidos por los diferentes gobiernos estatales y autonómicos.

En este sentido, Sandulli y Córdova (2014) señalan que las oficinas de farmacia en España se encuentran sometidas a fuerzas del mercado y regulatorias que están poniendo en duda la sostenibilidad del modelo actual.

Además, el observatorio de los medicamentos de la Federación Empresarial de Farmacéuticos Españoles (2017), señala que la situación económica de muchas farmacias ha empeorado en los últimos años, y algunas se han visto obligadas, incluso, a cerrar.

Por lo tanto, el propietario de una oficina de farmacia debe ser consciente de que los cambios que se han producido en estos últimos años en el sector han mermado significativamente sus ingresos y los beneficios obtenidos, hasta el punto de que en ocasiones se hace inviable el desarrollo de la actividad.

En este contexto, el objetivo para la oficina de farmacia es intentar hacerse menos dependiente de la receta de medicamentos y ampliar su facturación en productos de parafarmacia y especialidades farmacéuticas publicitarias (EFP), es decir, la venta libre.

Para alcanzar este objetivo, la política de recursos humanos debe ser un aspecto prioritario para el farmacéutico titular, que debe crear un entorno seguro de trabajo que propicie la flexibilidad y la innovación, definiendo objetivos y estrategias claras, comunicándolas a sus colaboradores y enseñándoles a asumir responsabilidades para alcanzar los objetivos propuestos (González, 2009).

En esta dirección, Díez y González (2011) señalan que la satisfacción del cliente depende en gran medida del equipo de colaboradores de la oficina de farmacia, que a través de su actitud y de la forma de relacionarse con el cliente, generará sensaciones positivas o negativas que modificarán el criterio del cliente en la compra y posible repetición de la compra. Además, la forma en que se atiende al cliente constituye un valor diferencial de la oficina de farmacia y contribuye a su fidelización.

Por lo tanto, con la finalidad de impulsar la venta de productos de parafarmacia y EFP, es fundamental para las farmacias disponer de personal cualificado, motivado y orientado a comprender y satisfacer las necesidades de sus clientes con el propósito de conseguir su fidelización.

En este sentido, el objetivo fundamental que se pretende con este trabajo de investigación exploratorio, es analizar la influencia que la orientación de los empleados a los clientes, la formación de los recursos humanos y los incentivos al personal de la farmacia ejercen sobre la venta libre, con la finalidad de compensar la pérdida de facturación obtenida 
a través de los medicamentos con receta y hacer a la farmacia mucho más competitiva y adaptada a los cambios del entorno.

Para conseguir este objetivo, se ha realizado un estudio de mercado a una muestra de 234 oficinas de farmacia de Galicia a través de una encuesta personal. Para el análisis de las relaciones entre las variables se empleó una regresión logística ordenada y la tabulación cruzada (prueba chi-cuadrado).

En el desarrollo del presente artículo se ha seguido la siguiente estructura. En primer lugar se analiza la situación actual de las oficinas de farmacia en España. En la segunda parte del trabajo se expone la importancia que tienen los recursos humanos para las oficinas de farmacia. A continuación se desarrolla el estudio empírico realizado y se presentan los resultados obtenidos. Finalmente, se exponen las conclusiones más importantes que pueden extraerse de los resultados obtenidos, así como las limitaciones del estudio y las futuras líneas de investigación.

\section{SITUACIÓN DE LAS OFICINAS DE FARMACIA EN ESPAÑA}

Las oficinas de farmacia son establecimientos sanitarios privados de interés público, sujetos a la planificación sanitaria que establezcan las Comunidades Autónomas. El farmacéutico titular-propietario de las mismas asistido por sus colaboradores, deberá prestar diferentes servicios básicos a la población en cuanto a medicamentos y productos sanitarios contenidos en la Ley 16/1997 de 25 de abril, de regulación de servicios de las Oficinas de Farmacia.

En la actualidad, la oficina de farmacia sufre un alto nivel de intervención por parte del Sistema Nacional de Salud, con constantes cambios legislativos que están poniendo en duda la sostenibilidad del modelo actual. Podemos señalar en esta dirección que en España desde 1997 se han redactado 29 leyes y cuatro Reales Decretos, estos últimos desde 2010, sobre el precio del medicamento, lo que ha provocado un descenso del margen neto de las farmacias hasta llegar a un 6,9 por 100 (Evolufarma, 2016).

Los recortes de márgenes de beneficio, la legislación restrictiva, la caída de los precios de los medicamentos, la llegada de otros competidores en el campo de la parafarmacia, el copago y la receta electrónica, la aparición de los medicamentos genéricos, unido a los aplazamientos de pago de la Seguridad Social y las corrientes liberalizadoras del sector que vienen de Europa, cuestionan que la calidad asistencial prestada hasta el momento por la oficina de farmacia sea sostenible.

Según López (2017), la facturación de las farmacias comunitarias en España se encuentra en una situación crítica, consecuencia de los diferentes cambios legislativos y reducciones de precios que se decretaron entre 2010 y 2012. Además, señala que otro factor fundamental que afecta a la viabilidad de las farmacias, ha sido el envío para su dispensación en los hospitales de la mayoría de las innovaciones farmacéuticas que generan un mercado 12 veces superior al que generan los medicamentos lanzados en las oficinas de farmacia.

En esta dirección, Sandulli y Córdova (2014) señalan que la principal caída en el volumen de negocio de las oficinas de farmacia es la reducción de la facturación al sistema nacional de seguridad social.

Como se puede observar en el siguiente cuadro, el gasto en medicamentos con receta creció hasta el año 2009, a partir de este año se produjo un descenso constante hasta llegar al año 2015 en que cambia la tendencia y se constata un ligero repunte, pero sin llegar al volumen de facturación de los años anteriores (pérdida del 11,43 por 100 desde 2007). En relación al número de recetas se puede observar que han aumentado un 6,91 por 100 respecto a 2007, sin embargo, el gasto por receta se redujo un 17,11 por 100. 


\section{CUADRO 1. EVOLUCIÓN DEL GASTO DE MEDICAMENTOS A TRAVÉS DE RECETA EN LAS OFICINAS DE FARMACIA EN ESPAÑA}

\begin{tabular}{|r|r|r|r|}
\hline Años & $\begin{array}{c}\text { Gasto (millones } \\
\text { de euros) }\end{array}$ & $\begin{array}{c}\text { Recetas } \\
\text { (millones) }\end{array}$ & \multicolumn{1}{c|}{$\begin{array}{c}\text { Gasto por } \\
\text { receta }\end{array}$} \\
\hline $\mathbf{2 0 0 7}$ & $11.192,2$ & 843,3 & 13,27 \\
\hline $\mathbf{2 0 0 8}$ & $11.971,2$ & 890,1 & 13,45 \\
\hline $\mathbf{2 0 0 9}$ & $12.506,9$ & 934,0 & 13,39 \\
\hline $\mathbf{2 0 1 0}$ & $12.201,6$ & 957,1 & 12,75 \\
\hline $\mathbf{2 0 1 1}$ & $11.144,1$ & 973,2 & 11,45 \\
\hline $\mathbf{2 0 1 2}$ & $9.773,4$ & 913,7 & 10,70 \\
\hline $\mathbf{2 0 1 3}$ & $9.183,2$ & 859,6 & 10,68 \\
\hline $\mathbf{2 0 1 4}$ & $9.360,5$ & 870,5 & 10,75 \\
\hline $\mathbf{2 0 1 5}$ & $9.535,1$ & 882,1 & 10,81 \\
\hline $\mathbf{2 0 1 6}$ & $9.912,9$ & 901,6 & 11,00 \\
\hline
\end{tabular}

Fuente: Elaboración propia a través de datos del Ministerio de Sanidad, Servicios Sociales e Igualdad.

Según el Observatorio de los medicamentos (2017), 975 oficinas de farmacia han pasado a ser subvencionas como consecuencia de la aplicación del Real Decreto Ley 16/2012, que introdujo la obligación de subvencionar a las farmacias que tuvieran unas ventas totales de menos de 200.000 euros anuales. Son las denominadas farmacias de viabilidad económica comprometida (VEC). De manera similar, Antares Consulting (2015) señala que el 3,4 por 100 de las farmacias entran dentro de la categoría VEC, un 20 por 100 se encuentran en una situación financiera desfavorable y un 5 por 100 con alto riesgo de liquidación de venderse de forma inadecuada.

Por otra parte, el consumo de medicamentos genéricos en España en 2016 representó el 20 por 100 del total del mercado farmacéutico en facturación y del 40 por 100 en unidades, cifras todavía muy inferiores a la media europea (25 por 100 y 60 por 100 respectivamente). Hay que tener en cuenta que esta situación repercute desfavorablemente en la facturación de las oficinas de farmacia, puesto que estos medicamentos aportan un ahorro considerable en la factura farmacéutica, reduciendo el coste efectivo entre un 40 y un 60 por 100 (Asociación Española de Medicamentos Genéricos, 2017). Asimismo el margen comercial de las farmacias se aplica sobre el precio de venta de los medicamentos, y por lo tanto, a menor precio menor beneficio.

Para hacer frente a estos cambios del entorno y conseguir que las farmacias sigan siendo rentables, los profesionales deben mantener una actitud proactiva y continuar innovando con la intención de sortear los posibles obstáculos que puedan afectar a la farmacia en el ámbito legislativo, comercial, de competencia o de cambios en el comportamiento de compra de los consumidores (De la Fuente, 2017a).

En este sentido, el farmacéutico debe asumir que su papel como gestor ha cambiado, ya no basta con dispensar un medicamento con receta, ahora la satisfacción del cliente requiere desarrollar una gran variedad de acciones como parte de la estrategia de marketing, buscando satisfacerlo y detectar sus necesidades futuras (De la Fuente, 2016a).

En esta situación, las farmacias tienen que asumir, si quieren seguir siendo competitivas y rentables, un cambio de actitud en la forma de gestionar tradicionalmente el negocio, en el que los farmacéuticos deben dejar de "despachar medicamentos" y adoptar una actitud más proactiva que partiendo del conocimiento de las necesidades y deseos de los clientes permita 
incrementar la venta libre. En esta dirección, los recursos humanos de las farmacias desempeñan un papel fundamental en la prestación de un servicio que satisfaga plenamente a los consumidores y constituya un instrumento fundamental de diferenciación en relación a sus principales competidores.

A pesar de lo expuesto anteriormente, las farmacias en España siguen dependiendo en gran medida de las ventas obtenidas de los medicamentos dispensados con receta médica. Según Besalduch y Besalduch (2017), "la farmacia tipo española” tiene una facturación de $500.000 €$ al año, de los cuales sólo un 24,95 por 100 procede de la venta libre (124.750€). Estas cifras son muy similares a las recogidas en los informes precedentes, lo que demuestra que a pesar de los cambios que se han producido en el sector, la farmacia sigue siendo muy dependiente de la venta de medicamentos con receta.

En este mismo informe se detallan algunas de las características fundamentales de estos establecimientos comerciales en relación a sus recursos humanos:

- La mayoría de las farmacias existentes en España son de un único titular (77,48 por 100), mientras que el 22,52 por 100 restante son de varios propietarios (comunidades de bienes o sociedades civiles).

- El peso de la mujer en las oficinas de farmacia es muy elevado, ya que la titularidad del 63,25 por 100 de las farmacias españolas corresponde a una mujer. Además, en el año 2015 se equilibra la cifra de los empleados en relación al sexo (51 por 100 mujeres), con lo que no existe tanta diferencia en relación con la contratación exclusiva del sexo femenino como en años anteriores.

- La plantilla media de una oficina de farmacia es de 3,37 trabajadores, lo que supone un ligero descenso en relación a años precedentes. La distribución de la plantilla media de la oficina de farmacia por categorías profesionales en el año 2015 es la siguiente: 1,65 de los empleados eran farmacéuticos, 1,50 auxiliar diplomado, 0,91 auxiliar, 0,49 mozo y 1,00 colaborador.

- El promedio de trabajadores con antigüedad inferior a 10 años es de 1,27, entre 10 y 25 años 1,40 y más de 25 años 1,46 años.

\section{LOS RECURSOS HUMANOS EN LAS OFICINAS DE FARMACIA}

En el ámbito de la dirección estratégica de la empresa, a partir de la década de los noventa, ha adquirido un gran protagonismo la denominada "Teoría de los Recursos y Capacidades”, que pone su énfasis en la importancia que poseen los recursos y capacidades de la empresa como fuente para obtener una ventaja competitiva sostenible que se traducirá en la obtención de mejores resultados empresariales (Barney, 1991).

La mayoría de los investigadores consideran que los recursos intangibles son los que proporcionan un mayor factor de diferenciación, ya que suelen ser recursos que han de nacer y desarrollarse dentro de la empresa y durante un cierto periodo de tiempo. Dentro de estos recursos, existe en los últimos años un cierto consenso en considerar los recursos asociados a las personas como uno de los elementos fundamentales del éxito de las organizaciones, debido a que son valiosos, imperfectamente imitables, escasos y limitadamente sustituibles (Danvila y Sastre, 2007).

Por otra parte, y desde una perspectiva de la evolución de la disciplina del marketing, surge el concepto de marketing interno, que tiene como objetivo fundamental motivar a todas las personas que colaboran con la empresa y conseguir su orientación al mercado.

El marketing interno comparte con la gestión de recursos humanos un aspecto fundamental que se resume en el objetivo de lograr que la organización posea trabajadores 
satisfechos y comprometidos, haciendo de éstos un recurso estratégico que contribuya a alcanzar los objetivos de la organización (Núñez, 2009).

Para Bohnenberger (2005), el marketing interno es una filosofía de gestión que tiene por objetivo una actuación en el ambiente externo de la organización (clientes), por medio de un ambiente de trabajo adecuado, que respete las necesidades y los deseos de los empleados, y con una orientación generalizada de todos los empleados para la satisfacción de los clientes de la empresa.

En este sentido, Alcaide (2008) considera que la gestión de los recursos humanos en una organización se debe basar en los siguientes principios:

- El capital humano es el activo más valioso de una empresa.

- El personal no es simplemente un recurso más de la organización, sino el único factor que puede generar ventajas competitivas a largo plazo.

- Los recursos humanos de una empresa influyen de forma directa y significativa en su rentabilidad.

- La creatividad, la capacidad de innovación, el potencial de crecimiento y de desarrollo futuro, la participación en el mercado y la estabilidad de una empresa dependen, sobre todo, de su capital humano.

En la actualidad las farmacias tienen diferentes instrumentos para intentar diferenciarse de sus competidores: surtido de productos de venta libre, servicios adicionales ofertados, localización del establecimiento comercial, diseño interior y exterior del punto de venta, etc., pero se puede considerar que uno de los factores que más influye en los clientes cuando eligen la farmacia en la que van a realizar sus compras es la atención recibida por los profesionales del establecimiento comercial.

Según González (2012), los recursos humanos que responden al perfil de la farmacia actual son una pieza clave en la rentabilidad de la misma, no basta con ser un realizador de tareas para las que se requieren unas determinadas capacidades, es necesario sobre todo que sean proveedores directos de la satisfacción al cliente integrado en un equipo competente.

El farmacéutico titular de una oficina de farmacia tiene que ser consciente de que los colaboradores de la farmacia forman parte de su equipo, y que sin ellos el día a día sería muy difícil llevarlo a cabo. Las personas son el activo más importante que una empresa puede tener. Sin las personas, sin su fidelidad, motivación y dedicación, lo máximo a que se puede aspirar es a la mediocridad. Cuestiones tales como si hay o no mucha clientela en el establecimiento, de qué tipo es o cómo se comporta, al igual que el número de vendedores, su apariencia física, vestimenta y actitud, influyen en la percepción global del establecimiento (Stein et al., 2010).

Por lo tanto, al igual que en cualquier otro tipo de empresas, la filosofía de apostar por el personal es básica en el sector de las farmacias. En este sentido, lo que verdaderamente supone una característica diferencial de unas farmacias con respecto a otras son los empleados. Su preparación, organización, implicación y proactividad son claves para que una oficina de farmacia esté bien posicionada respecto a sus competidores (De la Fuente, 2013a). Además, un buen equipo profesional preparado y motivado es la mejor garantía de una excelente atención farmacéutica y de un aumento significativo de las ventas.

Teniendo en cuenta lo expuesto anteriormente, en esta investigación consideramos dentro de los recursos humanos como principales factores que pueden contribuir a la diferenciación de las oficinas de farmacia y por lo tanto a mejorar sus resultados, los siguientes elementos: (1) la orientación que los empleados tienen hacia los clientes, puesto que permitirá conocer mejor sus necesidades y conseguir su satisfacción y lealtad; (2) la 
formación, ya que aumenta las habilidades y competencia de los empleados para atender mejor a los clientes, además de contribuir a su crecimiento y satisfacción profesional; (3) la motivación e incentivos a los empleados, como instrumento que favorece su compromiso con el trabajo y contribuye a mejorar los resultados de la farmacia.

\subsection{Orientación de los empleados de la oficina de farmacia hacia el cliente}

Valenzuela et al. (2010) y Stock (2016) señalan que la orientación de las empresas a los clientes es una estrategia fundamental para conseguir clientes satisfechos y leales, y lograr que la empresa obtenga una ventaja competitiva que le permita incrementar su volumen de ventas.

El compromiso de la empresa con sus consumidores, materializado en el desarrollo de relaciones continuas con sus clientes aunque no generen ingresos inmediatos para la empresa, ayuda a reforzar su imagen y pueden influir en futuras decisiones de compra de los consumidores, lo que podría aumentar la rentabilidad y el valor del negocio a largo plazo. En este sentido, los empleados que están en contacto directo con los clientes desempeñan un papel fundamental ya que a menudo actúan como representantes de la empresa y desempeñan un papel activo en la prestación del servicio, proporcionando información, aconsejando en el uso del producto, recibiendo las quejas o reclamaciones, etc. (Cambra et al., 2014).

Por lo tanto, una de las claves del éxito de las empresas es su orientación hacia los clientes, intentando ofrecerles el mejor servicio posible en relación a las empresas competidoras. Uno de los aspectos fundamentales de esta orientación al mercado está en la capacidad del personal de la organización, sobre todo el que está en contacto con los clientes, de analizar las necesidades de los consumidores para realizarles una oferta que satisfaga sus expectativas y establecer relaciones estables y duraderas con ellos.

En esta dirección, el personal de atención al público representa el auténtico protagonista de la relación con el cliente en la oficina de farmacia, matizando, concretando y terminando la experiencia de compra. La comunicación del personal de atención al público constituye un factor primordial de coherencia con el espacio y ambiente del punto de venta, de la eficacia vendedora y del éxito empresarial de la farmacia (Ribera, 2013a).

Según Stein et al. (2010), la forma en que el equipo de colaboradores de la farmacia recibe al cliente, lo trata y le informa, es el valor diferencial esencial y el motor principal de la fidelización. Hay que sonreír, mirar al cliente a los ojos desde el mismo momento que entra por la puerta, cuidar las formas y la entonación y usar un lenguaje lo más comprensible posible, buscando siempre la satisfacción de los consumidores.

Por ello es muy importante que el personal vendedor de la farmacia se ponga en el lugar del cliente, de manera que su problema/necesidad se convierta en la suya propia (Ribera, 2013b).

En el sector farmacéutico, Soto (2010a) considera que hay que diferenciar entre pacientes y clientes de una oficina de farmacia. Paciente es la persona que acude a la farmacia para adquirir un medicamento o producto sanitario y cliente es la persona que acude a la farmacia para adquirir productos de parafarmacia.

Cuando el paciente demanda un medicamento el papel que debe asumir el empleado de la oficina de farmacia se debe basar en dos premisas fundamentales (Soto, 2010a):

- Actitud siempre activa durante la dispensación, cooperando con el médico y otros profesionales sanitarios a fin de conseguir resultados que mejoren la calidad de vida del paciente. 
- Asunción de responsabilidad profesional sobre el resultado del uso del medicamento en todos los actos de la dispensación y no sólo en aquellos casos en que el paciente requiere una consulta. El paciente debe sentir que la farmacia es un centro de consulta y de seguimiento sobre su medicamento y su patología, complementario al prestado en otras instancias sanitarias.

Por otro lado, cuando el cliente demanda un producto de parafarmacia, la estrategia de comunicación del empleado de la farmacia se debe basar en dos puntos fundamentales (Soto, 2010b):

- La actitud referida al consejo debe ser continuamente proactiva. El empleado debe interesarse siempre por las necesidades del consumidor, independientemente del nivel de conocimiento que éste tenga sobre los productos ofertados.

- Todo producto vendido o consultado por el cliente debe ser acompañado por parte del farmacéutico de un consejo sobre sus cualidades, su uso o sus interacciones.

Uno de los objetivos principales que debe perseguir una farmacia a través de la atención dispensada por los empleados a los pacientes/clientes debe ser su fidelización. En este contexto, Díez (2011), considera que las farmacias deben contemplar a los pacientes como una oportunidad y que tienen la "suerte" de que la enfermedad leve o la receta les obliga a entrar en la farmacia, lo cual supone una gran ventaja frente a otros establecimientos competidores. Pero se debe establecer con los pacientes/clientes una relación personalizada, duradera y consistente con él, condicionada por sus peculiaridades, independientemente de quien lo atienda la calidad debe ser siempre homogénea.

En sentido, De La Fuente (2016b) señala que los empleados tienen que apoyar las estrategias de marketing promovidas por la oficina de farmacia, con el objetivo de crear vínculos personalizados con los clientes, alejándose de los aspectos más comerciales y mejorando la experiencia de compra de los consumidores.

Por otra parte, el personal vendedor además de jugar un papel esencial desde el punto de vista comercial, se revela como uno de los factores más importantes de la animación del establecimiento comercial, ya que son la cara humana del punto de venta y en muchos casos generadores de sensaciones táctiles muy valoradas por los clientes (Díez et al., 2006).

Esto se engloba dentro de la comunicación neurolingüística, es decir, un proceso de comunicación basado en percepciones corporales emitidas y percibidas entre el cliente y el personal de atención en la oficina de farmacia, las cuales se producen de forma espontánea e inconsciente. Además, este lenguaje resulta, en muchas ocasiones, determinante en las decisiones de compra, y donde el uniforme del personal es un elemento muy importante para transmitir imágenes de marca y servicio al cliente (Dermoactiva, 2009).

Por lo tanto, considerando que cuando los empleados desarrollan un comportamiento orientado hacia los clientes contribuyen a su satisfacción y al incremento de las ventas de la empresa (Valenzuela et al., 2010; Homburg et al., 2011; Stock, 2016), se propone la siguiente hipótesis de investigación:

$\mathrm{H}_{1}$ : Existe una relación positiva entre la orientación de los empleados hacia los clientes y la venta libre de la oficina de farmacia.

\subsection{Formación de los empleados de la oficina de farmacia}

Debido a la rapidez con que cambian las habilidades y conocimientos requeridos y a la consideración de los empleados como un recurso competitivo esencial para obtener una ventaja competitiva duradera, la formación constituye uno de los factores esenciales que determinan la eficacia de las acciones realizadas en las organizaciones (Danvila, 2004). 
Según Fernández y Junquera (2013), la formación del empleado es un conjunto de actividades que permiten mejorar su rendimiento presente o futuro, aumentando su competencia laboral y mejorando su actitud hacia el trabajo.

Por otra parte, la formación debe considerarse como una inversión a medio y largo plazo y no como un gasto, ya que puede considerarse una fuente directa de productividad y competitividad de la empresa y un requisito básico del fenómeno empresarial y sinónimo de educación y adiestramiento (Danvila, 2004).

Además, una mayor y mejor formación sirve no sólo para mejorar la competitividad de la empresa y el servicio al cliente, sino también para incrementar la motivación de los empleados, impulsar su crecimiento profesional y contribuir a mejorar su satisfacción tanto a nivel profesional como personal. Contar con una plantilla de empleados cualificada y formada regularmente es el mayor activo de una organización moderna que quiera sobresalir en el mercado (De Ansorena, 2008).

Para De La Fuente (2017b), la formación es proporcionar a los empleados de la farmacia conocimientos en materia sanitaria, de comunicación y marketing, puesto que sólo una combinación de los tres conseguirá resultados efectivos. Cuanto mayor sea el grado de formación y preparación del equipo, mayor será su nivel de productividad, cualitativa y cuantitativamente.

Un aspecto fundamental que se debe tener en cuenta en la formación del personal de la oficina de farmacia está relacionado con la concienciación de los colaboradores de una transición de la mera dispensación del medicamento hacia el consejo farmacéutico. Cada empleado debe ser consciente de que el consejo farmacéutico debería estar presente en cada una de las operaciones comerciales que se realizan en la oficina de farmacia (medicamentos con o sin receta y productos de parafarmacia). Además, la formación constituye un paso fundamental para conseguir la implicación del equipo colaborador, de modo que sea capaz de potenciar la venta libre y los servicios como valor diferencial frente al cliente (Valero, 2014).

Valero (2014) señala que el personal representa la segunda partida más elevada de $\operatorname{costes}^{1}$ dentro de la cuenta de explotación de la oficina de farmacia. Por lo que se debería de invertir más en la formación de los recursos humanos para poder satisfacer plenamente a los clientes, ya que el personal es la herramienta fundamental de ventas que será capaz de dar salida a los productos que estratégicamente se hayan decidido incluir en el surtido del establecimiento (De La Fuente, 2014).

La formación debe ser planificada por el titular de la oficina de farmacia, de tal modo que la formación no solo sea continua y coherente, sino también un ciclo al que los empleados se acostumbran y un vehículo para su motivación. La formación que se debe proporcionar a los empleados se puede dividir en tres tipos (De La Fuente, 2017b):

- Formación puntual. Centra el conocimiento en determinados aspectos relacionados con el desarrollo de la farmacia y suscita interés por la relevancia y/o aplicación que pueda tener en el día a día.

- Formación continuada. La idea de formar a los empleados con constancia supone aportar herramientas para el ejercicio eficaz de la profesión, a la par que se incrementa el potencial de la farmacia mediante el perfeccionamiento y actualización del personal que se verá preparado y motivado para progresar. Además, se eleva el compromiso del empleado con el cumplimento de los objetivos y el bienestar del paciente.

\footnotetext{
${ }^{1}$ De acuerdo con Besalduch y Besalduch (2017) la partida de gastos de personal varía dependiendo de la facturación de la oficina de farmacia, este puede oscilar entre un 9,01 por 100 y un 11,66 por 100 sobre el volumen de ventas.
} 
- Formación de competencias. Con la evolución del rol del farmacéutico, se hace necesario formar al equipo para el desarrollo de determinados aspectos que favorezcan la creación de la marca de la farmacia, generar vínculos emocionales y personales con los clientes y proporcionar experiencias de compra diferentes.

Por lo tanto, el titular de la oficina de farmacia debe establecer un plan de formación dirigido a los empleados para que dispongan de los conocimientos y habilidades necesarias para aconsejar en todo momento a los clientes, fomentando la venta de productos relacionados con la parafarmacia y las especialidades farmacéuticas publicitarias (EFP) y promoviendo su fidelización.

En este contexto, Valero (2014) señala que la formación en diferentes áreas de negocio de la oficina de farmacia muestra un incremento considerable en el ticket medio de compra, así como en el número de operaciones de la misma, provocando un aumento de la facturación y del peso de la parafarmacia en el mix de venta y, por lo tanto, un aumento en la rentabilidad del negocio.

Así, las capacidades y competencias de los empleados son fundamentales para el éxito de la empresa. En este sentido, diversos estudios coinciden en señalar que la formación de los empleados contribuye a mejorar los resultados de la empresa (Danvila, 2004; Marín, 2009; Aragón y Esteban, 2010). En consecuencia, se propone la siguiente hipótesis de investigación:

$\mathrm{H}_{2}$ : Existe una relación positiva entre la formación de los empleados y la venta libre de la oficina de farmacia.

\subsection{Motivación e incentivos a los empleados de la oficina de farmacia}

Lograr que las personas den lo mejor de sí en su trabajo, incluso en circunstancias difíciles, es uno de los desafíos más permanentes y complicados a los que se enfrentan los directivos de las empresas (Nohria, et al., 2008).

Los directivos de las empresas deben analizar cuáles son los factores que motivan y contribuyen a la satisfacción de los trabajadores de la organización, desarrollando estrategias que enriquezcan su trabajo y estimulen su motivación. Las empresas necesitan empleados comprometidos y que se impliquen en el trabajo que deben desarrollar, más allá de su compromiso contractual, con el objetivo de alcanzar, a través de su satisfacción dentro de la organización, mejores resultados empresariales.

En este sentido, para Alcaide (2008), se debe tener en cuenta que el personal de la empresa tiene necesidades emocionales, de pertenencia, de sentirse apoyado, de que puede confiar en el desarrollo de sus conocimientos y habilidades, de sentir que tiene ante sí una carrera que puede desarrollar dentro de la organización y que ésta reconoce sus méritos de forma justa.

En el sector de la oficina de farmacia, De la Fuente (2013b) señala que la motivación de los empleados empuja a alcanzar metas personales en la vida y proporciona los alicientes necesarios para desarrollar las capacidades personales y profesionales. Conseguir equipos motivados e implicados en la venta supone un gran éxito en la gestión de la farmacia.

Existen diferentes instrumentos a disposición de los directivos de las empresas para motivar e incentivar a los empleados: recibir reconocimientos verbales o escritos, reconocimientos de tipo material (viajes, equipamiento del hogar, ayuda a la matrícula escolar de los hijos...), reconocimientos relacionados con el ambiente de trabajo, ascensos o

promoción dentro del organigrama de la empresa e incentivos económicos, entre otros. Éstos últimos son los más analizados en la literatura relacionada con los incentivos percibidos por los trabajadores de las empresas porque tradicionalmente fueron los más utilizados por las 
organizaciones, aunque en la actualidad también tienen gran relevancia los basados en los beneficios sociales de los trabajadores.

Los incentivos vinculados al rendimiento buscan mejorar el beneficio global de la organización, posibilitando la atracción y retención de empleados más productivos e incitando a los empleados a aumentar eficientemente su esfuerzo.

Tradicionalmente se considera que las retribuciones percibidas por los empleados de la empresa en función de una serie de objetivos previamente establecidos, influyen directamente en el desempeño de su actividad dentro de la organización y, por lo tanto, en los resultados empresariales. Sin embargo, Verano et al., (2006) y Dessler (2009) señalan que diferentes investigaciones ponen en duda que, a pesar de la importancia que tiene esta práctica retributiva en las empresas, estos incentivos económicos influyan en el rendimiento de los empleados y en los resultados de la organización.

Además, el pago de incentivos tiene un fundamento psicológico, por lo que no todas las personas reaccionan de la misma forma ante una retribución y no todas las retribuciones son adecuadas para todas las situaciones (Dessler, 2009).

En cualquier caso, en la actualidad, la mayoría de las empresas utiliza los sistemas retributivos como un elemento motivador para alcanzar los objetivos previstos. Dentro de estos sistemas cobra especial relevancia en los últimos tiempos los que se basan en una distribución entre fijo y variable, considerándose esta como la retribución idónea, siempre y cuando los objetivos y la forma de valorarlos estén correctamente fijados.

El titular de la farmacia, como director comercial del negocio, es el responsable de todos los aspectos relativos al rendimiento y la eficacia de las actuaciones comerciales que se llevan a cabo. Además de definir los objetivos a conseguir, planificar el conjunto de estrategias a implantar y organizar su ejecución, debe encargarse de que el equipo esté adecuadamente formado y motivado. Siendo consciente que con unos medios muy limitados, es importante definir claramente el papel que debe desempeñar cada empleado en la estrategia perseguida (Godás, 2007).

Por lo tanto, es muy importante para las empresas motivar a sus empleados, especialmente los que están en contacto directo con los clientes, con el objetivo de mejorar su rendimiento y el de la organización. En este sentido, diversas investigaciones (Verano, 2003; Miao, et al., 2007) consideran que incentivar económicamente a los empleados puede contribuir al incremento de las ventas de la empresa. En consecuencia se propone la siguiente hipótesis de investigación:

$\mathrm{H}_{3}$ : Existe una relación positiva entre los incentivos económicos recibidos por los empleados y la venta libre de la oficina de farmacia.

\section{ESTUDIO EMPÍRICO}

Este trabajo pretende ser una primera aproximación a la importancia que tienen los recursos humanos en una oficina de farmacia como instrumento fundamental que ayuda a reforzar la imagen de la empresa, contribuye a la satisfacción y fidelización de los clientes actuales, influye en las decisiones de compra de otros consumidores no habituales y, en definitiva, contribuye a la diferenciación del negocio y a obtener una ventaja competitiva sostenible que permita a las farmacias incrementar la venta libre.

Como se ha expuesto anteriormente, el objetivo principal que se pretende con esta investigación es analizar la influencia que la orientación de los empleados de la oficina de farmacia, su formación y los incentivos promovidos por los titulares de la farmacia ejercen sobre la venta libre. 
Además, considerando que los empleados de la farmacia constituyen un factor fundamental de animación del punto de venta y de creación de imagen del establecimiento comercial, se analizaron diversos aspectos relacionados con su indumentaria.

\subsection{Medida de las variables}

En esta investigación se han analizado cuatro aspectos relacionados con los recursos humanos de la oficina de farmacia: la orientación de los empleados hacia los clientes, la formación de los empleados, la existencia de incentivos económicos para los empleados y la imagen de los empleados en el punto de venta.

La formación y los incentivos económicos de los empleados se basan en las respuestas aportadas por el titular de la oficina de farmacia, utilizando para ello una variable dicotómica:

La farmacia organiza cursos de formación dirigidos a sus empleados $\square$ SI $\square$ NO

La farmacia tiene desarrollado un programa de incentivos económicos dirigido a sus empleados $\square$ SI $\square$ NO

Por otra parte, para analizar la orientación de los empleados de la farmacia hacia los clientes, se plantearon tres cuestiones respondidas por los visitadores de farmacia, empleando una escala de Likert de 1 a 5, (1=Totalmente en desacuerdo y 5= Totalmente de acuerdo):

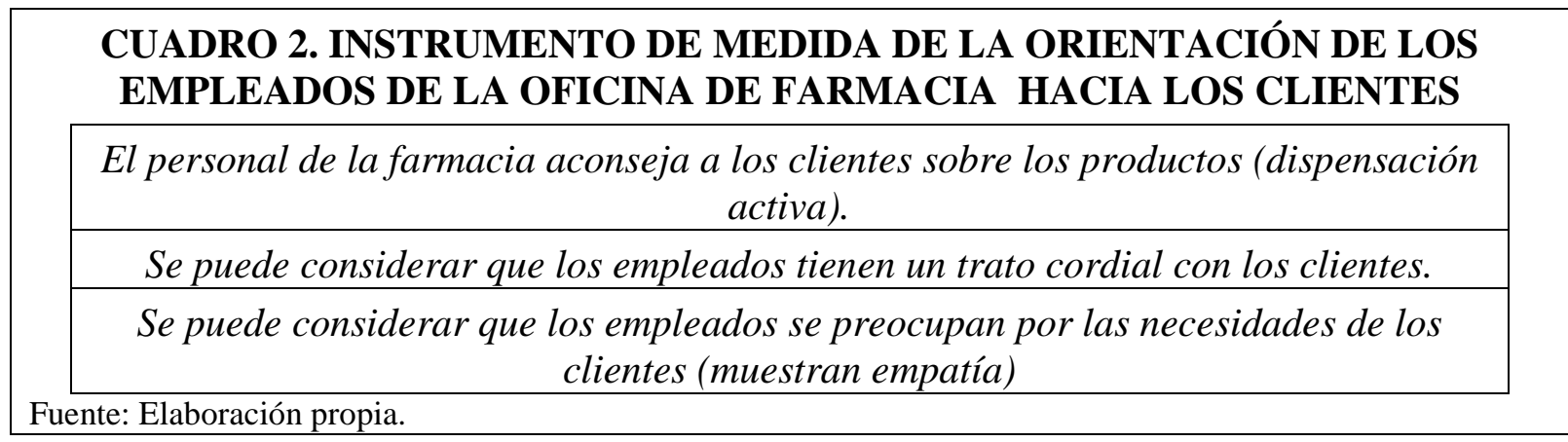

Considerando que los empleados de la oficina de farmacia que están en contacto con los clientes constituyen un factor fundamental en la creación de la imagen del establecimiento comercial y contribuyen a la percepción que el cliente puede tener sobre la calidad del servicio prestado, se han contemplado en esta investigación diversos aspectos relacionados con el uniforme utilizado por los empleados de la farmacia. Además, se debe tener en cuenta que la farmacia es un establecimiento dedicado a la venta de productos y servicios de salud, por lo que es muy importante tener especial cuidado con la apariencia del personal vendedor. En este sentido se plantearon las siguientes preguntas dicotómicas respondidas por los visitadores de farmacia:

\section{CUADRO 3. INSTRUMENTO DE MEDIDA DE LA INDUMENTARIA DE LOS EMPLEADOS DE LA OFICINA DE FARMACIA}

\begin{tabular}{|lrr|}
\hline El personal tiene un distintivo con su nombre & $\square$ SI & $\square$ NO \\
\hline El uniforme del personal es el fijado por la farmacia con su logotipo & $\square$ SI & $\square$ NO \\
\hline El uniforme de los empleados está limpio & $\square$ SI & $\square$ NO \\
\hline
\end{tabular}

Fuente: Elaboración propia. 
Finalmente, para medir las ventas de las oficinas de farmacia de productos de parafarmacia y especialidades farmacéuticas publicitarias se utilizó la siguiente escala:

\section{CUADRO 4. INSTRUMENTO DE MEDIDA DE LA VENTA LIBRE EN LA OFICINA DE FARMACIA}

\begin{tabular}{l} 
Indique cuál es el volumen de ventas obtenido por su farmacia de productos de \\
parafarmacia y EFP en el último año: \\
\hline Menos de 150.000 euros. \\
\hline Entre 150.001 y 300.000 euros. \\
\hline Más de 300.000 euros. \\
\hline
\end{tabular}

Fuente: Elaboración propia.

\subsection{Definición de la población y diseño de la muestra}

La población objeto de estudio de la presente investigación la constituyen todas las oficinas de farmacia con sede en la Comunidad Autónoma de Galicia. Según el Consejo general de colegios oficiales de farmacéuticos, en diciembre de 2013 había en Galicia 1.345 oficinas de farmacia, las cuales constituyen inicialmente la población objeto de estudio.

La recogida y cumplimentación de los datos, fue realizada por cinco visitadores de farmacia de la Cooperativa Farmacéutica Española (COFARES) de sus delegaciones de Galicia.

Aunque COFARES es la empresa líder en distribución de medicamentos en España, no es proveedora de todas las farmacias de España y de Galicia. Concretamente en Galicia hay 505 farmacias a las que suministran diferentes productos relacionados con el sector farmacéutico. Por lo tanto, esta es la población objeto del presente estudio, la cual representa el 37,55 por 100 de la totalidad de las oficinas de farmacia existentes en Galicia. Se debe señalar que no existe ninguna condición por parte de la empresa para que cualquier farmacia de Galicia entre a formar parte de sus clientes. Por consiguiente, se puede considerar que estas 505 farmacias son representativas de las farmacias gallegas. La ficha técnica del estudio se presenta en el siguiente cuadro

\section{CUADRO 5. FICHA TÉCNICA DEL ESTUDIO}

\begin{tabular}{|l|l|}
\hline Universo & 1.345 oficinas de farmacia \\
\hline Población objeto de estudio & 505 oficinas de farmacia \\
\hline Ámbito & Comunidad Autónoma de Galicia \\
\hline Tamaño muestral & $\begin{array}{l}\text { 234 oficinas de farmacia (46,34\% de la población objeto } \\
\text { de estudio) }\end{array}$ \\
\hline Procedimiento de muestreo & Muestreo no probabilístico discrecional \\
\hline Trabajo de campo & Marzo y Abril de 2014 \\
\hline $\begin{array}{l}\text { Preguntas respondidas por los } \\
\text { visitadores de Farmacia }\end{array}$ & $\begin{array}{l}\text { - Orientación de los empleados hacia los clientes } \\
\text { - Indumentaria de los empleados }\end{array}$ \\
\hline $\begin{array}{l}\text { Preguntas respondida por los } \\
\text { titulares de las oficinas de } \\
\text { farmacia }\end{array}$ & $\begin{array}{l}\text { - Formación de los empleados } \\
\text { - Programa de incentivos económicos de la farmacia } \\
\text { - Venta libre de la farmacia }\end{array}$ \\
\hline
\end{tabular}

Fuente: Elaboración propia. 
Una vez analizados los cuestionarios recibidos, se detectó que 53 farmacias no contestaron a la pregunta relacionada con las ventas obtenidas en el último año en productos de venta libre.

Este problema se tuvo en cuenta cuando se realizó el análisis de los datos. En este sentido para todos los análisis descriptivos realizados se consideraron la totalidad de los cuestionarios obtenidos, mientras que para la verificación de las hipótesis planteadas se utilizaron 181 cuestionarios.

\subsection{Procedimientos y técnicas de análisis de los datos} Windows.

Para el tratamiento de los datos se utilizó el paquete estadístico SPSS 20.0 para

Para realizar los análisis descriptivos de las variables se calcularon las frecuencias y las medias de las variables incluidas en la investigación.

Para contrastar las hipótesis, teniendo en cuenta las características de la variable orientación de los empleados a los clientes, se utilizó una regresión logística ordenada que nos permite comprobar si existe relación entre las variables y si esta relación es positiva o negativa. También se utilizó la prueba chi-cuadrado para verificar las otras dos hipótesis planteadas.

\subsection{Estudio descriptivo de la muestra}

En este apartado expondremos las características más significativas de la muestra en relación a los recursos humanos de las farmacias y a la venta libre.

En relación a la orientación de los empleados hacia los clientes, como se puede observar en la figura 1, las farmacias que formaron parte de la encuesta tienen un personal altamente implicado con los clientes, obteniendo la mayor puntuación media $(4,30)$ en el trato que les dispensan. Este es un aspecto muy importante que favorece la venta libre y la fidelización de los clientes.

FIGURA 1. ORIENTACIÓN DE LOS EMPLEADOS DE LA OFICINA DE FARMACIA HACIA LOS CLIENTES

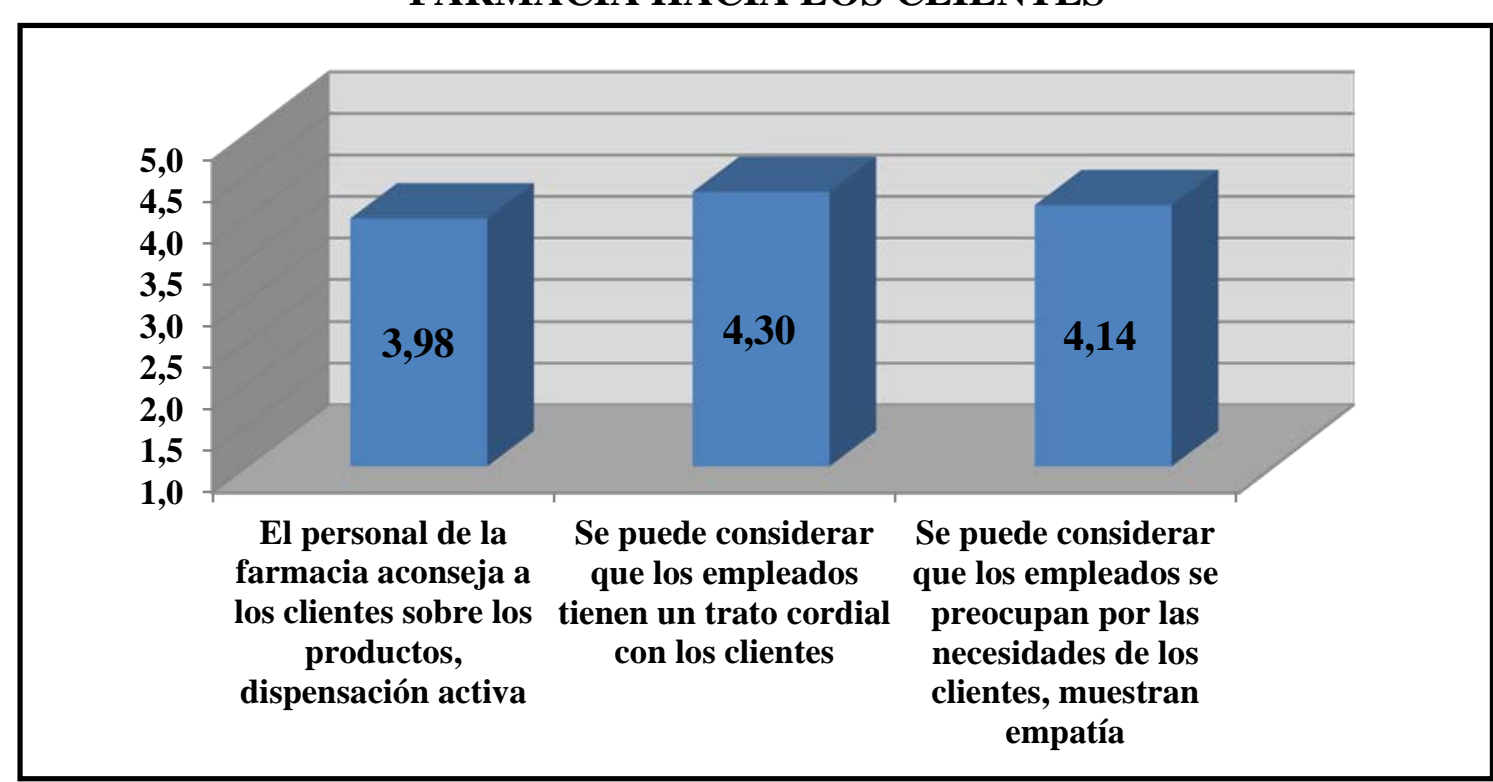

Fuente: Elaboración propia. 
Por otra parte, el personal de la farmacia juega un papel esencial en la animación del establecimiento comercial y en la imagen que esta quiere transmitir a sus clientes. En este sentido, un aspecto fundamental que se debe cuidar es la apariencia del personal vendedor.

En el cuadro 4, se comprueba que en la mayoría de las farmacias de Galicia el personal lleva un distintivo con su nombre (81,50 por 100). Sin embargo, un aspecto negativo que se debe destacar, es que la mayoría de ellas no proporciona a sus empleados el uniforme con el logotipo de la farmacia (30,60 por 100). Consideramos que es un aspecto que deberían tener en cuenta las farmacias con el objetivo de transmitir una imagen homogénea ante sus clientes. También se constata que en general el uniforme de los empleados está limpio, como no podría ser de otra forma teniendo en cuenta el tipo de comercio minorista que estamos analizando.

Por otra parte, a pesar de la importancia que le conceden las investigaciones a la formación de los empleados como factor clave para conseguir una mayor motivación y satisfacción del personal y que dispongan de los conocimientos necesarios para poder atender a los pacientes/clientes de la farmacia, menos del 50 por 100 proporciona formación a sus empleados.

Finalmente, se puede observar que son muy pocas las farmacias de Galicia que tienen planificado un programa de incentivos económicos para conseguir empleados ampliamente motivados y que den lo mejor de sí en su trabajo (35,50 por 100).

\section{CUADRO 6. IMAGEN, FORMACIÓN E INCENTIVOS A LOS EMPLEADOS DE LA OFICINA DE FARMACIA}

\begin{tabular}{|l|c|c|}
\hline $\begin{array}{l}\text { Imagen, formación e incentivos a los empleados de la } \\
\text { farmacia }\end{array}$ & SI & NO \\
\hline El personal tiene un distintivo con su nombre & $81,50 \%$ & $18,50 \%$ \\
\hline $\begin{array}{l}\text { El uniforme del personal es el fijado por la farmacia, con su } \\
\text { logotipo }\end{array}$ & $30,60 \%$ & $69,40 \%$ \\
\hline El uniforme de los empleados está limpio & $93,10 \%$ & $6,90 \%$ \\
\hline $\begin{array}{l}\text { La farmacia organiza cursos de formación dirigidos a sus } \\
\text { empleados }\end{array}$ & $49,40 \%$ & $50,60 \%$ \\
\hline $\begin{array}{l}\text { La farmacia tiene desarrollado un programa de incentivos para } \\
\text { sus empleados }\end{array}$ & $35,50 \%$ & $64,50 \%$ \\
\hline
\end{tabular}

Fuente: Elaboración propia.

Como se expuso anteriormente, de las 234 farmacias encuestadas, 181 respondieron a la pregunta relacionada con las ventas obtenidas en el último año en productos de venta libre.

Los resultados obtenidos se recogen en el siguiente cuadro. Como se puede observar, la mayoría de las farmacias de Galicia tienen una facturación en venta libre inferior a 300.000 euros $(75,20$ por 100$)$. Siendo las más representativas las que tienen una facturación inferior a 150.000 euros (38,70 por 100$)$.

\section{CUADRO 7. VENTA LIBRE DE LAS OFICINAS DE FARMACIA}

\begin{tabular}{|l|r|}
\hline \multicolumn{1}{|c|}{$\begin{array}{c}\text { Volumen de ventas obtenido por la farmacia en productos de } \\
\text { parafarmacia y EFP en el último año }\end{array}$} & Porcentaje \\
\hline Menos de 150.000 euros & $38,70 \%$ \\
\hline Entre 150.001 y 300.000 euros & $36,50 \%$ \\
\hline Más de 300.000 euros & $24,80 \%$ \\
\hline
\end{tabular}




\subsection{Resultados de la investigación}

A continuación se procede al análisis de las hipótesis planteadas. En el caso del análisis de la relación entre la orientación de los empleados a los clientes y la venta libre, debemos señalar que los coeficientes obtenidos del análisis de regresión logística ordenada, indican la probabilidad logarítmica ordinada para un aumento de una unidad en la puntuación de la variable dependiente, es decir que si aumenta una unidad la puntuación media de cada variable, el coeficiente indica la probabilidad de que la venta libre pase a estar en una categoría superior.

\subsubsection{Relación entre la orientación de los empleados hacia los clientes y la venta libre de la oficina de farmacia}

Teniendo en cuenta la naturaleza exploratoria de la presente investigación, se creó una nueva variable de naturaleza latente, es decir, que no puede observarse de forma directa y que su medición se realiza a través de variables observadas o manifiestas.

Para la creación de esta variable latente seguimos la recomendación de Bollen (1989), que considera necesario generar un batería de ítems o indicadores a través de los que se representarán las diferentes variables latentes.

Estas variables son de naturaleza reflectiva, es decir, las variables latentes observables son expresadas en función del constructo (Bollen, 1989). Su característica clave es que un cambio en la variable latente se verá reflejado en un cambio en todos los indicadores (Bollen y Lennox, 1991). Por lo tanto, las variables manifiestas u observables son las variables dependientes, mientras que la variable latente es la independiente (Diamantopoulos, 1999). Las medidas de un constructo reflectivo deberían estar correlacionadas y alcanzar una alta consistencia interna, elevado valor del Alpha de Cronbach (Roldán, 2000).

Se considera que el valor mínimo que debe tener el Alpha de Cronbach es 0,7. Sin embargo, si el objetivo del estudio es de carácter confirmatorio no deberían aceptarse valores inferiores a 0,8 (Luque, 1997).

Se procedió al cálculo de este coeficiente y se comprobó que la consistencia interna de la orientación de los empleados, evaluada a través del Alpha de Cronbach, es muy elevada $(0,898)$, superando el valor máximo recomendado por Luque (1997).

El análisis efectuado demuestra la existencia de una relación significativa $(p=0,005)$ y positiva (coeficiente $=0,509$ ) entre la orientación de los empleados hacia los clientes y la venta libre.

Por lo tanto, contar con un equipo de profesionales preocupado por la satisfacción de las necesidades de sus clientes, proporcionándoles información y aconsejándoles acerca de los productos que pueden satisfacer sus necesidades, escuchando sus quejas o reclamaciones, es decir, con una clara orientación hacia al cliente, contribuye a incrementar la venta libre de la oficina de farmacia.

En esta investigación se confirma que una de las claves del éxito de los negocios es la orientación de la empresa hacia los clientes. Como señala Ribera (2013a), el personal de atención al público representa el auténtico protagonista de la relación con el cliente en la oficina de farmacia, constituyendo un factor primordial de coherencia con el espacio y ambiente del punto de venta, de la eficacia vendedora y del éxito empresarial de la farmacia. 


\subsubsection{Relación entre la formación de los empleados y la venta libre de la oficina de}

\section{farmacia}

El resultado obtenido demuestra la existencia de una relación positiva y estadísticamente significativa entre los cursos de formación recibidos por los empleados de la oficina de farmacia y la venta libre $(\mathrm{p}=0,017)$.

Las farmacias que facilitan y motivan a sus empleados a adquirir una mayor formación se ve reflejada en las ventas de productos de parafarmacia y EFP. Como se puede observar, el 70,80 por 100 de las farmacias que diseñan cursos de formación para sus colaboradores, tienen unas ventas libres superiores a 150.000 euros. Por el contrario, el 48,40 por 100 de las farmacias que no fomentan la formación entre sus empleados facturan menos de 150.000 euros. Este resultado coincide con la mayoría de las investigaciones realizadas en el ámbito de los recursos humanos que señalan que la formación de los empleados es muy importante, no sólo para incrementar las ventas de la empresa, sino también como instrumento de motivación y de mejora de su actitud hacia el trabajo.

\section{CUADRO 8. RELACIÓN ENTRE LA FORMACIÓN DE LOS EMPLEADOS Y LA VENTA LIBRE DE LA FARMACIA.}

\begin{tabular}{|c|c|r|r|r|r|}
\hline & & \multicolumn{4}{|c|}{ Venta libre de la oficina de farmacia } \\
\cline { 3 - 6 } $\mathbf{X}^{\mathbf{2}}(\mathbf{\alpha = 0 , 0 5 )}$ & $\mathbf{p}=\mathbf{0 , 0 1 7}$ & $\begin{array}{c}\text { Menos de } \\
\mathbf{1 5 0 . 0 0 0 €}\end{array}$ & $\begin{array}{c}\text { Entre } \\
\mathbf{1 5 0 . 0 0 1} \\
\mathbf{3 0 0 . 0 0 0} \mathbf{~}\end{array}$ & $\begin{array}{c}\text { Más de } \\
\mathbf{3 0 0 . 0 0 0 €}\end{array}$ & \multirow{2}{*}{ Total } \\
\hline $\begin{array}{c}\text { Formación de los } \\
\text { empleados }\end{array}$ & No & $48,40 \%$ & $34,10 \%$ & $17,50 \%$ & $100 \%$ \\
\cline { 2 - 6 } & $\mathrm{Si}$ & $29,20 \%$ & $39,30 \%$ & $31,50 \%$ & $100 \%$ \\
\hline
\end{tabular}

Fuente: Elaboración propia.

4.5.3. Relación entre la existencia de incentivos económicos percibidos por los empleados y la venta libre de la oficina de farmacia

Los resultados obtenidos revelan la existencia de una relación positiva y estadísticamente significativa entre los incentivos económicos percibidos por los empleados y la venta libre $(\mathrm{p}=0,008)$.

Como se puede observar en el cuadro 7 , el 72,80 por 100 de las farmacias que tienen desarrollado un programa de incentivos económicos dirigidos a sus empleados facturan más de 150.000 euros, mientras que el 54,40 por 100 de las que no lo poseen superan esa cifra de ventas. Por lo tanto, estos resultados coinciden con otras investigaciones en señalar que los incentivos económicos percibidos por los empleados favorecen la obtención de unos mejores resultados empresariales.

\section{CUADRO 9. RELACIÓN ENTRE LOS INCENTIVOS ECONÓMICOS PERCIBIDOS POR LOS EMPLEADOS Y LA VENTA LIBRE DE LA FARMACIA.}

\begin{tabular}{|c|c|c|c|c|c|}
\hline \multirow[b]{2}{*}{$X^{2}(\alpha=0,05)$} & \multirow[b]{2}{*}{$p=0,008$} & \multicolumn{4}{|c|}{ Venta libre de la oficina de farmacia } \\
\hline & & $\begin{array}{l}\text { Menos de } \\
150.000 €\end{array}$ & $\begin{array}{c}\text { Entre } 150.001 € y \\
300.000 €\end{array}$ & $\begin{array}{c}\text { Más de } \\
300.000 €\end{array}$ & Total \\
\hline \multirow{2}{*}{$\begin{array}{c}\text { Incentivos } \\
\text { económicos para los } \\
\text { empleados } \\
\end{array}$} & No & $45,60 \%$ & $36,80 \%$ & $17,60 \%$ & $100 \%$ \\
\hline & $\mathrm{Si}$ & $27,20 \%$ & $36,40 \%$ & $36,40 \%$ & $100 \%$ \\
\hline
\end{tabular}

Fuente: Elaboración propia. 


\section{DISCUSIÓN}

\subsection{Conclusiones}

Los numerosos cambios legislativos que se han realizado en el sector farmacéutico, los cambios en el comportamiento de los consumidores, la alta competitividad del mercado, la situación de crisis económica que ha atravesado nuestro país en los últimos años, entre otros factores, han propiciado una reducción considerable del volumen de ventas y beneficios de las oficinas de farmacia que ponen en duda la viabilidad comercial de muchas de ellas.

En el año 2016 de las casi 22.000 farmacias existentes en España, 975 se consideraban de viabilidad económica comprometida (Observatorio de los medicamentos, 2017). Además, en un período de 10 años las farmacias han perdido más de un 11 por 100 en la facturación de medicamentos con receta (Ministerio de Sanidad, Servicios Sociales e Igualdad, 2017), los cuales representan aproximadamente el 75 por 100 de una farmacia tipo (Besalduch y Besalduch, 2017).

En esta situación, los recursos humanos de las farmacias desempeñan un papel fundamental en la prestación de un servicio que satisfaga plenamente a los consumidores y permita compensar la pérdida de facturación de los medicamentos con receta con la venta libre.

En esta investigación se ha analizado la influencia que la orientación hacia los clientes, la formación y los incentivos recibidos por los empleados de la farmacia tienen en la venta libre. Las principales conclusiones que se pueden extraer de los análisis realizados son las siguientes:

En primer lugar, se comprobó que la orientación de los empleados a los clientes es bastante elevada entre las farmacias analizadas (con puntuaciones alrededor de 4 en todos los indicadores), y que esta implicación de los empleados en la satisfacción de las necesidades de los clientes contribuye a incrementar la venta libre.

En segundo lugar, se constata que menos del 50 por 100 de las oficinas de farmacia organiza cursos de formación dirigidos a sus empleados, y que solamente un tercio de las farmacias encuestadas tienen desarrollado un programa de incentivos para motivar a sus colaboradores. Además, se ha demostrado que estos dos factores tienen una influencia bastante significativa en la venta libre de la farmacia.

Finalmente, se comprobó que la mayoría de las oficinas de farmacia concede una gran importancia a la indumentaria utilizada por los empleados, con la excepción de que el uniforme no es fijado por la farmacia, ya que puede contribuir de manera importante a la creación de imagen de marca de la farmacia.

\subsection{Implicaciones para la gestión}

La situación que vive actualmente la farmacia, la obliga a transformarse hacia un modelo más competitivo, con unos establecimientos comerciales más eficientes, rentables y mejor gestionados. El farmacéutico titular debe estar dispuesto a evolucionar para garantizar la subsistencia de un modelo en el que el mayor beneficiado debe ser el paciente, por lo que debe jugar un papel sanitario, junto a otros profesionales, en la prevención, atención farmacéutica y mantenimiento de la salud.

Además, los importantes cambios que se han producido en España en los últimos años en este sector han supuesto una reducción considerable de las ventas de medicamentos con receta, por lo que se ve obligada a compensar la caída de ingresos obtenidos en la venta de 
medicamentos por prescripción facultativa, con el aumento en la facturación de productos de venta libre.

En esta dirección, consideramos fundamental para obtener éxito en el desarrollo de este nuevo modelo, dotar a la farmacia de una estructura organizativa empresarial dirigida por un farmacéutico titular que asuma el liderazgo de la gestión, con un planteamiento claro de los objetivos que se quieren conseguir, y con un plan de formación continua que mantenga al equipo de colaboradores motivado y preparado para ofrecer un servicio de calidad, lo que influirá positivamente en la satisfacción de los consumidores y en la creación de una imagen de una farmacia preocupada por la bienestar de sus clientes.

Para mejorar el servicio prestado, la comunicación con los clientes, su satisfacción y fidelización, es fundamental que los colaboradores adopten una actitud positiva y proactiva hacia los clientes, informándoles y aconsejándoles en la compra de los productos que necesitan. Este cambio de actitud en la gestión del negocio contribuirá positivamente en la imagen de la farmacia y en el aumento de los ingresos, sobre todo a través de la venta de productos de parafarmacia y EFP.

Para conseguir empleados comprometidos con su trabajo, el farmacéutico titular debe diseñar estrategias que enriquezcan su trabajo y fomenten su motivación. Entre los instrumentos más utilizados para motivar e incentivar a los empleados destacan especialmente las recompensas económicas, que influyen positivamente en el incremento de las ventas de la farmacia.

Finalmente, se debe señalar la importancia de desarrollar programas de formación continua dirigidos a los empleados, para que dispongan de los conocimientos y habilidades necesarias para prestar un servicio de calidad y fomentar la venta de productos relacionados con la parafarmacia y EFP. Además, la formación también constituye un instrumento fundamental para conseguir la implicación del equipo colaborador.

\subsection{Limitaciones y futuras líneas de investigación}

Los resultados presentados en este trabajo deben interpretarse con precaución, ya que esta investigación no está exenta de una serie de limitaciones que deben tenerse en cuenta a la hora de evaluar y generalizar las conclusiones obtenidas. En todo caso, las limitaciones apuntadas pueden ser el germen de futuras investigaciones relacionadas con la gestión de los recursos humanos en las farmacias. Estas limitaciones son las siguientes:

En la muestra se incluyen exclusivamente farmacias pertenecientes a la comunidad autónoma de Galicia lo cual dificulta la generalización de los resultados obtenidos a otros ámbitos geográficos.

En la investigación realizada faltan determinadas cuestiones relacionadas con los recursos humanos como: el proceso de selección del personal, las competencias y habilidades de los empleados, el liderazgo del titular de la farmacia, los requerimientos del puesto de trabajo, entre otras.

Al tratarse de un estudio exploratorio, somos conscientes de que en futuras investigaciones sería necesario mejorar las escalas de medida empleadas así como los instrumentos de análisis utilizados para contrastar las hipótesis planteadas.

Finalmente, teniendo en cuenta que el estudio fue realizado por cinco profesionales pertenecientes al equipo comercial de COFARES, y aunque se explicaron previamente las instrucciones que deberían seguir para cubrir la parte del cuestionario que les correspondía, somos conscientes que las respuestas a las preguntas podían variar de un sujeto a otro debido a la diferente percepción que puede tener cada participante ante una mismo hecho o cuestión. 


\section{BIBLIOGRAFÍA}

Alcaide, J.C. (2008): “Los tres enfoques clave del marketing interno”, Harvard Deusto Marketing y Ventas, $\mathrm{n}^{\circ}$ 87, pp. 72-79.

Antares Consulting (2015): Situación y cifras claves de la farmacia en 2014. Informe, disponible en http://www.antares-healthlines.com/nc/articulo/situacion-y-cifrasclaves-de-la-farmacia-en-2014/

Aragón, A. y Esteban, N. (2010): "La formación de la empresa española: ¿sólo se busca mejorar los resultados organizacionales?”, Universia Business Review, vol. 2, pp. 3556.

Asociación Española de Medicamentos Genéricos (2017): Genéricos en España: Aún lejos de la media europea, disponible en http://www.aeseg.es/es/medicamentos-genericos-enespana.

Barney, J.B. (1991): "Firm resources and sustained competitive advantage”, Journal of Management, vol. 17, $\mathrm{n}^{\mathrm{o}}$ 1, pp. 99-120.

Besalduch, J.M. y Besalduch, X. (Coords.)(2017): Informe Anual Aspime de Oficinas de Farmacia 2017, XVII edición, Ed. Elsevier España, S.L.U, Barcelona.

Bohnenberger, M.C. (2005): Marketing interno: La actuación conjunta de recursos humanos y marketing en busca del compromiso organizacional, Tesis Doctoral, Universitat de les Illes Balears.

Bollen, K. (1989): Structural equations with latent variables, Ed. John Wiley \& Sons, Inc., Nueva York.

Bollen, K. y Lennox, R. (1991): “Conventional Wisdom on measurement: a structural equation perspective”, Psychological Paradigm, vol. 110, n² 2, pp. 305-314.

Cambra, J.; Melero, I. y Vázquez, I. (2014): “The role of frontline employees in customer engagement”, Revista Española de Investigación de Marketing ESIC, vol. 18, pp. 6777.

Danvila, I. (2004): La generación de capital humano a través de la formación, un análisis de su efecto sobre los resultados empresariales, Tesis Doctoral, Universidad Complutense de Madrid.

Danvila, I. y Sastre, M.A. (2007): “Capital humano y ventaja competitiva sostenible: un análisis de la relación entre la formación y los resultados empresariales”, EsicMarket, $n^{0} 128$, pp. 145-187.

De Ansorena, S. (2008): "La formación como elemento clave para ser una empresa competente”, Capital Humano, n 219, pp. 72-77.

De La Fuente, L. (2013a): “Negociación con proveedores: buenas prácticas”, Farmacia Profesional, vol. 27, $\mathrm{n}^{\mathrm{0}}$ 2, pp. 19-22.

De La Fuente, L. (2013b): De la A a la Z en la gestión de la oficina de farmacia, Ed. Club de la farmacia, Madrid.

De La Fuente, L. (2014): Despierta farmacia, Ed. Club de la Farmacia, Madrid.

De La Fuente, L. (2016a): “Acuerdos win to win entre farmacia e industria”, Farmacia Profesional, vol. 30, $\mathrm{n}^{\circ}$ 6, pp. 10-12.

De La Fuente, L. (2016b): “Importancia del 'sell out' en la farmacia”, Farmacia Profesional, vol. 30, no 4, pp. 11-13.

De La Fuente, L. (2017a): “Situación y panorama de la farmacia 2016”, Farmacia profesional, vol. 31, $\mathrm{n}^{\mathrm{0}}$ 1, pp. 10-12. 
De La Fuente, L. (2017b): “La formación de los empleados: ¿gasto o inversión?”, El farmacéutico, disponible en http://elfarmaceutico.es/index.php/revista-elfarmaceutico-el-farmaceutico-joven/item/7985-la-formacion-de-los-empleados-gastoo-inversion\#.WTg9Hk21v5o.

Dermoactiva (2009): Gestión comercial. Potenciar promociones, animaciones y campañas, Ed. Vichy y el Consejo General de Colegios Oficiales de Farmacéuticos, Madrid.

Dessler, G. (2009): Administración de recursos humanos, 13ª edición, Ed. Pearson, México.

Diamantopoulus, A. (1999): "Viewpoint. Export performance measurement: reflective versus formative indicators”, International Marketing Review, vol. 16, n 6, pp. 444-457.

Diez, E. C.; Landa, F. J. y Navarro, A. (2006): Merchandising: teoría y práctica, $2^{\text {a }}$ Edición, Ed. Pirámide, Madrid.

Díez, M.L. (2011): "El cliente del siglo XXI: importancia de su conocimiento”, Farmacia Profesional, vol. 25, n¹, pp. 27-31.

Díez, M.L. y González, A. (2011): “La fidelización: cuestión de emociones”, Farmacia Profesional, vol. 25, n ${ }^{\circ}$, pp. 27-31.

Evolufarma (2016): Segundo estudio de la presentación digital de la farmacia en España 2016, Ed. Evolufarma, Madrid.

Fernández, E. y Junquera, B. (2013): Iniciación a los recursos humanos, Ed. Septem Ediciones, Oviedo.

Godás, L. (2007): “Ámbito farmacéutico”, Gestión farmacéutica, vol. 26, nº 6, pp. 106-110.

González, A. (2012): Manual Práctico de Gestión de la Oficina de Farmacia. Gestión del día a día en tiempo de crisis, Ed. AMV Ediciones, Madrid.

González. M.A. (2009): “¿Sabemos dirigir?”, Farmacia Profesional, vol. 23, n 2, pp. 24-26.

Homburg, C.; Müller, M. y Klarmann, M. (2011): "When should the customer really be king? On the optimum level of salesperson customer orientation in sales encounters", Journal of Marketing, vol. 75, pp. 55-74.

Ley 16/1997 de 25 de abril, de Regulación de Servicios de las Oficina de Farmacia. Boletín Oficial del Estado, n 100 de 26 de abril 1997.

López, J.M. (2017): “Facturación de la farmacia: Una situación insostenible”, disponible en https://www.diariofarma.com/2017/01/26/facturacion-la-farmacia-una-situacioninsostenible.

Luque, T. (1997): Investigación de marketing, Ed. Ariel, Barcelona.

Marín, M.L. (2009): La formación de la empresa, comportamiento y resultados. El caso de las grandes empresas en Cataluña, Tesis Doctoral, Universidad Politécnica de Cataluña.

Miao, C.F.; Evans, K.R. y Zou, S. (2007): “The role of salesperson motivation in sales control systems-intrinsic and extrinsic motivation revisited”, Journal of Business Research, vol. 60, pp. 417-425.

Ministerio de Sanidad, Servicios Sociales e Igualdad (2017): Facturación de recetas médicas, Disponible en https://www.msssi.gob.es/profesionales/farmacia/datos/home.htm

Nohria, N.; Groysberg, B. y Lee, L.E. (2008): “Motivación de los empleados: un poderoso modelo nuevo", Harvard Business Review, vol. 86, nº 7, pp. 84-91.

Núñez, J.M. (2009): Revisión conceptual del marketing interno y verificación de la utilidad de sus principios fundamentales en la empresa, Tesis Doctoral, Universidad de la Laguna. 
Observatorio de los medicamentos (2017): "El consumo de medicamentos no recuperará los valores de 2009 hasta dentro de siete años”, Disponible en http://fefe.com/2017/observatorio-medicamento/consumo-medicamentos-ventamercado-farmaciavalores-2009-2023/.

Real Decreto-Ley 16/2012, de 20 de abril, de medidas urgentes para garantizar la sostenibilidad del Sistema Nacional de Salud y mejorar la calidad y seguridad de sus prestaciones. Boletín Oficial del Estado, n ${ }^{\circ} 98$ de 24 de abril de 2012.

Ribera, C. (2013a): “Organización del punto de venta en la farmacia. ¿Cómo hacerlo?”, Revista Acofar pp. 1-5, disponible en http://www.revistaacofar.com/revista/ 19/07/2013.

Ribera, C. (2013b): Técnicas de venta. Merchandising y técnica de venta en la oficina de farmacia, Ed. EdikaMed, S.L., Barcelona.

Roldán, J.L. (2000): Sistemas de información ejecutivos (EIS): Génesis, implantación y repercusiones organizativas, Tesis Doctoral, Universidad de Sevilla.

Sandulli, F. (Dir.) y Córdova, D. (Coord.) (2014): El sistema retributivo de la farmacia española: Evaluación de modelos fallidos y propuestas eficaces, Ed. Vinces Consuling, Madrid.

Soto, A. (2010a): “Comunicación con el cliente/paciente (I). El paciente”, Farmacia Profesional, vol. 24, n ${ }^{\circ}$ 1, pp. 24-28.

Soto, A. (2010b): “Comunicación con el cliente/paciente (II). El cliente”, Farmacia Profesional, vol. 24, $\mathrm{n}^{\circ}$ 2, pp. 28-32.

Stein, M.V.; Tejera, C.; Fortuny, S.; Fisselier, X. y Arias, A. (2010): Cómo convertir tu farmacia en un súper proyecto, Ed. Profit, Barcelona.

Stock, R.M. (2016): "Understanding the relationship between frontline employee boreout and customer orientation”, Journal of Business Research, vol. 69, n 10, pp. 4259-4268.

Valenzuela, L.M.; Mulki, J.P. y Jaramillo, J.F. (2010): "Impact of customer orientation, inducements and ethics on loyalty to the firm: customers'perspective", Journal of Business Ethics, vol. 93, pp. 277-291.

Valero, P. (2014): "Formación del equipo en la farmacia”, Farmacia Profesional, vol. 28, no 6, pp. 15-17.

Verano, D. (2003): La influencia de la retribución variable en el rendimiento de la empresa: Una aplicación empírica al diseño de la retribución para la fuerza de ventas, Tesis Doctoral Universidad de las Palmas de Gran Canaria.

Verano, D.; Melián, S. y García, J.M. (2006): "Retribución variable para equipos de ventas: ¿Es la teoría de costes de transacción un marco válido para diseñar sistemas de incentivos eficaces?”, Investigaciones Europeas de Dirección y Economía de la Empresa, vol. 12, n 2, pp. 35-54. 